\title{
Fabry Disease: from Diagnosis to Therapy
}

\author{
Ifrah Shafqat ${ }^{1}$ and Dr. Shaheen Shahzad ${ }^{1}$, \\ ${ }^{I}$ (Department of Biotechnology and Bioinformatics, International Islamic University Islamabad, Pakistan)
}

\begin{abstract}
Fabry disease (FD) is an accelerating, $X$-linked hereditary disorder of glycosphingolipid metabolism which occurs due to improper lysosomal $\alpha$-galactosidase A activity. Its pan-ethnic and the annually reported occurrence may underestimate the true frequency of the disease. Classically affected hemizygous males, with no residual $\alpha$-galactosidase A activity may display all the characteristics like cardiovascular (cardiomyopathy), cutaneous (angiokeratoma), renal (kidney failure), neurological (pain), cerebrovascular and cochleo-vestibular signs of the disease while heterozygous females have similar symptoms ranging from very mild to severe. Incomplete action of lysosomal $\alpha$-galactosidase A results in gradual increase of globotriaosylceramide within lysosomes, supposed to set off a flow of cellular events. Enzyme analysis may occasionally help to detect heterozygote but in females results are often questionable because of random X-chromosomal inactivation that obligates the molecular testing (genotyping). Due to ethical reasons determination of enzyme activity through prenatal diagnosis is carried out only in male's fetus. The subsistence of atypical variants and the unavailability of a specific therapy complicate the genetic counseling. Enzyme replacement therapy (ERT) is the only specific therapeutic option that introduced recombinant human $\alpha$-galactosidase A. End stage renal disease and critical cardiovascular or cerebrovascular complications limit the life-expectancy of untreated males and females. Oral therapy drives the research forward into active site specific chaperones. Adjunctive therapy of ERT with chemical chaperone therapy can arrest disease succession.
\end{abstract}

Keywords: $\alpha$-Galactosidase A (AGAL), chaperon therapy, fabry disease, glycoshingolipids, lysosomal storage disease

\section{Introduction}

Fabry disease (FD) (OMIM: 301500) comparatively less prevalent in females is characterized by angiokeratoma corporis diffusum (skin abnormality), cornea verticillata, hypohidrosis (excessive sweating), gastroenteritis, obstructed airflow, improper kidney functioning and premature myocardial infractions [1]. The occurrence of clinical symptoms may vary in intensity and frequency in different patients. It is an X-linked inborn error of $\alpha$-galactosidase A deficiency (AGAL). The multisymptomatic FD with its complex manifestations has slow progression of symptoms with age. The main cause of death is heart or renal failure. The Fabry Outcome Survey (FOS) data showed that patients experience neuropathic pain with burning sensation in the soles and palms. Pain attacks in joints and abdomen was also reported. The intensity of pain increases with fever, physical exercise and alcohol. Specific facial appearance with prominent lips thickening, frontal bossing and supraorbital ridges in hemizygous males of 12 to 14 years of age were also observed [1]. Angiokeratoma that is characterized by red, small, raised spots appear slowly in the areas of buttocks, and inner thighs. Some of the male patients become intolerant to exercise with reduced production of saliva and tears. Rare cases report hyperhidrosis at early age [2,3]. Sensory organs specifically eyes and ear are affected by FD. Cornea verticillata (opacity) and hearing loss is most obvious [4]. Other severe symptoms include air flow obstruction, asthma dementia, learning difficulty, and depression [1,5].

Atypical variants of FD manifest cardiac and renal involvement having AGAL activity between 2-20\% than normal [6]. Cardiac involvements in hemizygous males include the defects of heart valve and conduction disturbance [7]. Low AGAL activity also found in FD patients of renal variants having mutation in galactosidase gene [8].

\section{$1.1 \alpha$ - Galactosidase A}

Fabry disease is actually caused by excessive accumulation of a class of lipids called glycoshingolipids, especially ceramidetrihexoside, or globotriasylceramide (Gb3) [9]. Sphingolipids have structural moiety of long chain aminoalcohol sphingosine Fig. 1. Second carbon atom two of long chain fatty acid sphingosine is bound to nitrogen atom and the structure formed is called ceramide. First carbon atom of ceramide portion of sphingosine is linked to varying numbers of sugars [10]. The structure formed is called glycosphingolipids. Glycosphingolipids (GSLs) are components of the plasma membrane. These components are degraded in the lysosome through endocytic pathway [11]. The catabolic pathway of GSLs needs different cofactors and enzymes. The AGAL enzyme in its active form requires saposin B to start its activity on substrate that is Gb3 [12]. The insufficient activity of AGAL to catalyze the hydrolytic cleavage of the terminal galactose from $\mathrm{Gb} 3$ 
causes the excessive accumulation of globotriaosylsphingosine (lyso-Gb3) in kidney, lungs and erythrocytes $[13,14]$. Lyso-Gb3 and Gb3 are cytotoxic and the toxicity of lyso-Gb3 is greater than Gb3 [15]. Sphingosine

$$
\begin{array}{r}
\mathrm{CH}_{3}-\left(\mathrm{CH}_{2}\right)_{12}-\mathrm{CH}=\mathrm{CH}-\mathrm{CH}-\mathrm{CH}-\mathrm{CHOH} \\
\mathrm{OH} \mathrm{NH}
\end{array}
$$

Ceramidetrihexoside/ globotriasylceramide $(\mathrm{Gb} 3)$

Sphingosine-Glucose-Galactose-Galactose

Globotriaosylsphingosine (lyso-Gb3)

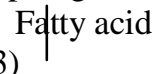

\section{Sphingosine-Glucose-Galactose-Galactose}

Fig. 1 The core structural moiety is long chain sphingosine [10].

\subsubsection{The Structure of Human a-galactosidase A}

The X-ray crystallography showed the homodimeric structure of AGAL, made up of two domains. The $\mathrm{N}$ - terminal domain contains the active site that is present at the $\mathrm{C}$ - terminal end of $\beta$-strands and second is $\beta$ sandwich of 8 anti-parallel $\beta$-strands at the C-terminal Fig. 2 [15]. The distance between two active sites is $50 \AA$ that remained same in ligand and free enzyme showing the independent functioning of both domains. Structurally $\alpha$-galactosidase A and $\alpha$-galactosidase B ( $\alpha$-N-acetylgalactosaminidase) have same structure but the absence of an 'N-acetyl recognition loop' in $\alpha$-galactosidase differs the substrate specificity of both enzymes [16]. The genes encoding $\alpha$-galactosidase A and $\alpha$-galactosidase B are localized on chromosomes Xq22.1 and 22q13, respectively. The genetically distinct locations further confirm the difference between two enzymes [17]. The reaction mechanism of enzyme carried out by double displacement reaction as the two nucleophiles attack on carbon of substrate (Gb3). D170 is the catalytic nucleophile and D231 is the catalytic base/acid in human AGAL [18].

\subsection{The Genetic Basis}

The homodimeric precursor $\alpha$-galactosidase A $(101 \mathrm{kD})$ is encoded by $12 \mathrm{~kb}$ gene present at $\mathrm{X}$ chromosome ( Xq22.1 region) have 429 amino acids, 7 exons ranging from 92 to 291 base pairs Fig. 3 [14, 19]. Patients with FD have not only the structural changes in AGAL enzyme but also slower heat inactivation as compared to normal enzyme. Over 599 mutations were recorded in defective galactosidase gene that is making the enzyme non functional [20,21,22]. Mutations causing FD include base substitutions in exons, splice site mutations, small insertions, large deletions and partial gene rearrangements [23]. Dissimilar mutations at different positions showed lack of mutational hot spot. Galactosidase gene rearrangements with five partial gene deletions of size 0.4 to $5.5 \mathrm{~kb}$ were detected by southern blot analysis. Four among the 5 deletions were in exon 2 containing Alu repeats [24]. Repetitive Alu elements are about $30 \%$ of the $12 \mathrm{~kb}$ gene but only 1 deletion in case of FD is resulted from Alu repeats recombination [25]. Eng and Desnick reviewed 31 point mutations, 15 rearrangements, and 3 mRNA processing defects [26]. Rare intronic splice site mutations and other twenty novel mutations were reported by Topaloglu while testing 30 unrelated families of FD [27]. At low pH only one exonic variant D313Y found in $0.45 \%$ of normal Caucasian which had reduced and lowered the plasma activity of enzyme [28]. Upstream region of exon 6 showed single point mutation in two Japanese patients with cardiac phenotype of FD [29]. Missense mutation in exon 3, new splice site mutation in consensus sequence of exon 4 resulted in loss of catalytic activity of related enzyme [30]. New mutations including 20 missense, 2 nonsense, 1 splice site, 8 insertions/ deletions in exon 7 and complex rearrangements in exon 1 and 2 were also reported by Christine and his colleagues [31]. Missense mutations were also reported in the renal variants of FD patients [8, 32].

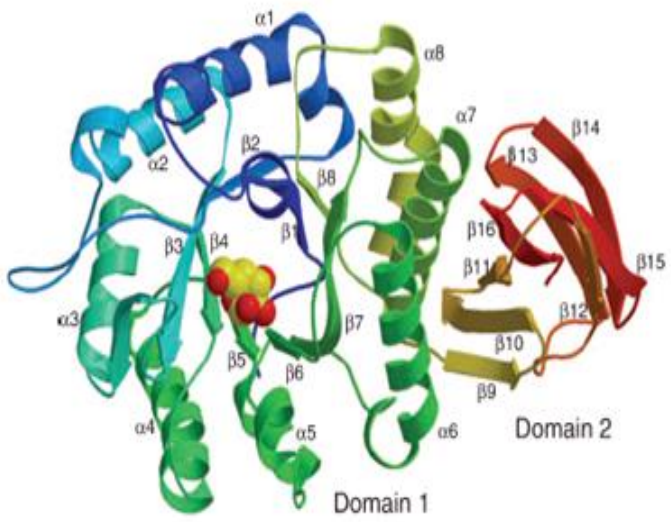

Fig. 2 Structure of AGAL monomer Domain 1 contains active-site; domain 2 contains antiparallel $\beta$ strands [15] 


\subsubsection{Diagnosis at Molecular Level}

The activity of AGAL in blood/ leukocytes can determine the affected patient. But mostly affected females show normal enzyme activity. So, the enzyme activity test is not the reliable testing for disease patient identification [33]. Another method for biochemical diagnosis is to check plasma Gb3 level that is comparatively lower in males even in the normal range [34]. Urinary Gb3 level is also unreliable option because Gb3 level will not increase in patients of late onset variants [35]. Sequence analysis of galactosidase gene identifies all the affected patients. Targeted mutational analysis is better option for the areas where the disease occurrence is high. Deletion testing detects the whole gene deletions in carrier/ heterozygous females. After the confirmation of disease family screening and pedigree analysis is effective to identify previous unrecognized affected individuals. Similarly genetic counseling before prenatal diagnosis can easily identify the risk level of associated symptoms but there are always unanswered ethical issues that limit the boundaries [36].

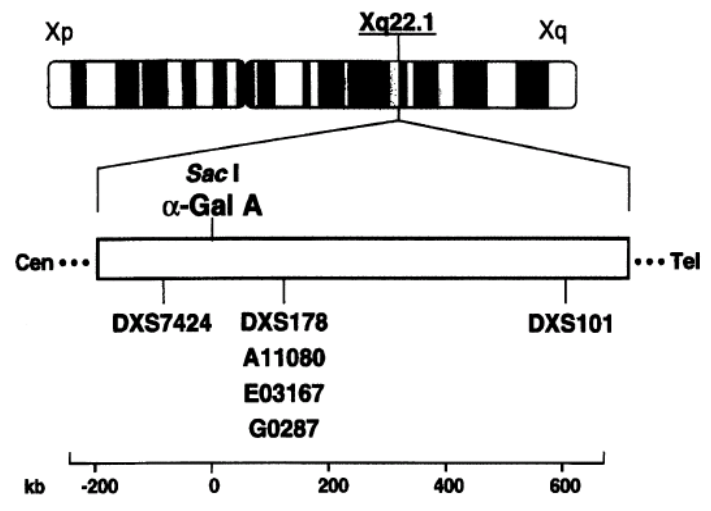

Fig. 3 Idiogram of the human G-banded X-chromosome (Xq22.1) with mapping of the genomic region flanking $\alpha$-Gal A indicating the relative positions of the markers used for haplotype analysis [19].

Looking at the penetrance and expressivity pattern of FD in males and females Dobyns and his coworker suggested using the term of X-linked trait instead of recessive or dominant one. Females are mosaic for some X-linked genes due to random transcriptional silencing of $\mathrm{X}$-chromosome known as $\mathrm{X}$ chromosome inactivation (XCI). Therefore carrier females range from no symptoms to the atypical ones. The patterns of X-inactivation in different organ systems in females justify the variability pattern of FD symptoms to some extent [37]. A study was carried out to determine the relation between mutant $\alpha$-galactosidase A allele and skewed X-inactivation. Highly skewed samples were tested for nonsense mutations in AGLA. Results showed no correlation between clinical signs, XCI ratios, age, and enzymatic activity of $\alpha$-galactosidase A [38].

\subsection{Therapy and Management of Fabry Disease}

Treatment of FD commonly starts with the symptoms management because of the involvement of almost all the organs. Loss of renal function delayed by antihypertensive and ACE (acetyl cholinesterase) inhibitors. Heart functioning is normalized by coronary bypass grafting, artificial pacemaker, and antiarrhythmic drugs $[39,40]$. But these are generalized treatments for dipping symptoms. Some specific treatments are discussed below

\subsubsection{Enzyme Replacement Therapy (ERT)}

The recombinant human AGLA introduced the disease specific enzyme replacement therapy (ERT). This therapy varies in different patients especially when it comes to children and females. In Europe agalsidase alfa and agalsidase beta are two commercially available enzyme preparations for FD treatment. Alfa galsidase alfa produced by human skin fibroblast culture and agalsidase beta produced by recombinant of human cDNA AGAL expression in Chinese Hamster Ovary (CHO) cells [41, 42]. The safety and efficacy of ERT is generalized by the reduction of disease severity of FD patients after one year of therapy with agalsidase alfa [43, 44]. In children the plasma Gb3 level along with GI symptoms were reduced after agalsidase beta therapy [45]. The enzyme replacement therapy has been implemented in clinical trials but it has limitations for long term trials [46].

\subsubsection{Small Molecular Drugs}

Small molecular drugs are $80-90 \%$ of marketed drugs and showed response quickly even after oral administration. Ability to cross blood-brain barrier, lack of autoimmune reaction, and lowest manufacturing cost favor these drugs therapeutically and economically [47]. Such type of drug therapy involves activation of 
residual enzyme through substrate reduction therapy (SRT), chemical chaperone therapy (CCT) and activation of AGAL promoter. Small AGAL promoter molecules enhances the transcription of mutated gene and results in increase of AGAL protein in lysosomes [48]. Similarly in case of residual enzyme activation, it is practically more complicated to design specific activator molecules of protein [49]. SRT and CCT are relatively better option as compare to other therapies.

\subsubsection{Substrate Reduction Therapy (SRT)}

Glucosylceramide synthase inhibitors (N-butyldeoxynojirimycin) lowered down the rate of $\mathrm{Gb} 3$ synthesis thus reduces the accumulation of globotriaosylceramide, glycosphingolipid in lysosomes [50]. Contradictions of N-butyldeoxynojirimycin are due to its non specificity for glucosylceramide synthetase resulted in gastrointestinal problems [51, 52]. Combination of ERT and SRT might improve treatment efficacy.

\subsubsection{Chemical Chaperon therapy (CCT)}

Missense mutation in FD results in the synthesis of catalytically active but unstable lysosomal protein. These mutated enzymes are retained and then degraded in endoplasmic reticulum because of their misfolded conformation [53]. By using active site specific chaperones, the stability and misfolding can be enhanced that will prevent the premature degradation by endoplasmic reticulum [54]. Basically chemical chaperons are small molecules that assist in correct folding and trafficking of mutated enzyme [55]. Clinical trial is carried out for deoxygalactonojirimycin (DGJ, marketed as Amigal TM by Amicus Therapeutics, Inc.) as a chemical chaperone therapy [56]. Co-administration of small pharmacological chaperone AT1001 improvers the stability and pharmacological properties of recombinant human AGAL by preventing denaturation at neutral $\mathrm{pH}$ and normal body temperature. In this way CCT enhances the efficiency of ERT [57]. Oral administration of these pharmacological chaperones support this therapy but unfortunately only $42 \%$ of genotype respond these so called small molecules [58].

\subsubsection{Gene Therapy}

The alternative method is gene therapy with a number of options for gene delivery system. Dissemination of AGAL transduced haematopoietic cells from Fabry mice have enzymatic correction of recipient cells that led to reduction in lipid storage [46]. Gene therapy usually target liver and skeletal muscles for the production of therapeutic proteins followed by systemic action. Recombinant AGAL administered into the blood through the lower respiratory tract via adenoviral vector. The resulted enzyme was expressed in the epithelium of respiratory tract and after diffusion enters into the circulatory system. In this way lungs are reported to be the viable organ for gene therapy [59]. Unavailability of reliable data for clinical trials of gene therapy is due to involvement of viral vectors [47].

\section{Conclusion}

The complexity of Fabry disease at genetic, molecular and diagnostic level makes it the most sophisticated disease not only at diagnostic level but also at therapeutic level. Discovery of new exonic and intronic mutations and lack of mutational hot spot results in reluctance of clinical trials of gene therapy. Therefore target of the physiologist is basically to lessen the associated symptoms to expand the life span of patient. Combination of ERT, SRT and CCT promises to increase the efficacy of FD at different stages of clinical trials. Development of cost effective and long term stable therapy would definitely be a breakthrough in the world of human genetics.

\section{References}

[1] K.D. MacDermot, A. Holmes, and A.H. Miners, Anderson-Fabry disease: Clinical manifestations and impact of disease in a cohort of 98 hemizygous males, Journal of Medical Genetics, 38(11), 2001, 750-760.

[2] W.J. Cable, E.H. Kolodny, and R.D. Adams, Fabry disease: impaired autonomic function, Neurology, 32(5), 1982, 498-502.

[3] M. Ries, U. Ramaswami, R. Parini, B. Lindblad, C. Whybra, I. Willers, A. Gal, and M. Beck, The early clinical phenotype of Fabry disease: a study on 35 European children and adolescents, European Journal of Pediatrics, 162(11), 2003, 767-772.

[4] D. Hajioff, Y. Enever, R. Quiney, J. Zuckerman, K. Mackermot, and A. Mehta, Hearing loss in Fabry disease: the effect of agalsidase alfa replacement therapy, Journal of Inherited Metabolic Disease, 26(8), 2003, 787-794.

[5] D.M. Rosenberg, V.J. Ferrans, J.D. Fulmer, B.R. Line, J.A. Barranger, and R.O. Brady, Chronic airflow obstruction in Fabry's disease, The American Journal of Medicine, 68(6), 1980, 898-905.

[6] S. Ishii, H.H. Chang, K. Kawasaki, K. Yasuda, H.L. Wu, S.C. Garman, and J.Q. Fan, Mutant alpha-galactosidase A enzymes identified in Fabry disease patients with residual enzyme activity: biochemical characterization and restoration of normal intracellular processing by 1- deoxygalactonojirimycin, The Biochemical Journal, 406(2), 2007, 285-295.

[7] B. Sachdev, T. Takenaka, H. Teraguchi, C. Tei, P. Lee, and W.J. McKenna, Prevalence of Anderson-Fabry disease in male patients with late onset hypertrophic cardiomyopathy, Circulation, 105(12), 2002, 1407-1411.

[8] S. Nakao, C. Kodama, T. Takenaka, A. Tanaka, Y. Yasumoto, A. Yoshida, T. Kanzaki, A.L. Enriquez, C.M. Eng, H. Tanaka, C. Tei, and R.J. Desnick, Fabry disease: detection of undiagnosed hemodialysis patients and identification of a "renal variant" phenotype, Kidney International, 64(3), 2003, 801-807. 
[9] C.C. Sweeley, and B. Klionsky, Fabrys disease: classification as a sphingolipidosis and partial characterization of a novel glycolipid, The Journal of Biological Chemistry, 238, 1963, 3148-3150.

[10] R.O. Brady, (1966), Sphingolipidoses, The New England Journal of Medicine, 275(6), 1966, $312-318$.

[11] T.D. Weibel, and R.O. Brady, Systematic approach to the diagnosis of lysosomal storage disorders, Mental Retardation and Developmental Disabilities Research Reviews, 7(3), 2001, 190-199.

[12] R. Kase, U. Bierfreund, A. Klein, T. Kolter, K. Itoh, and M. Suzuki, Only sphingolipid activator protein B (SAP-B or saposin B) stimulates the degradation of globotriaosylceramide by recombinant human lysosomal $\alpha$-galactosidase in a detergent-free liposomal system, FEBS Letters, 393(1), 1996, 74-76.

[13] R.O. Brady, A.E. Gal, R.M. Bradley, E. Martensson, A.L. Warshaw, and L. Laster, Enzymatic defect in Fabry disease ceramidetrihexosidase deficiency, The New England Journal of Medicine, 276(21), 1967, 1163-1167.

[14] R.J. Desnick, Y.A. Ioannou, and C.M. Eng, Galactosidase A deficiency: Fabry disease, in C.R. Scriver, A.L. Beaudet, and W.S. Sly, D. Valle (Ed.), The Online Metabolic and Molecular Bases of Inherited Diseases, in (New York: McGraw-Hill, 2001 ) $3733-3774$.

[15] U. Schueler, T. Kolter, C.R. Kaneski, J.K. Blusztajn, M. Herkenham, K. Sandhoff, and R.O. Brady, Toxicity of glucosylsphingosine (glucopsychosine) to cultured neuronal cells: a model system for assessing neuronal damage in Gaucher disease Type 2 and 3 , Neurobiology of Disease, 14(3), 2003, 595-601.

[16] S.C. Garman, and D.N. Garboczi, The molecular defect leading to Fabry disease: structure of human $\alpha$-galactosidase, Journal of Molecular Biology, 337(2), 2004, 319-335.

[17] A.M. Wang, and R.J. Desnick, Structural organization and complete sequence of the human $\alpha$-N-acetylgalactosaminidase gene: homology with the $\alpha$-galactosidase A gene provides evidence for evolution from a common ancestral gene, Genomics, 10(1), 1991, $133-142$

[18] A.I. Guce, N.E. Clark, E.N. Salgado, D.R. Ivanen, A.A. Kulminskaya, H. Brumer, and S.C. Garman, Catalytic mechanism of human alpha-galactosidase, The Journal of Biological Chemistry, 285(6), 2010, 3625-3632.

[19] M. Caggana, G.A. Ashley, R.J. Desnick, and C.M. Eng, (1997). Fabry disease: molecular carrier detection and prenatal diagnosis by analysis of closely linked polymorphisms at Xq22.1, American Journal of Medical Genetics, 71(3), 1997, $329-335$.

[20] G. Romeo, and B.R. Migeon, Genetic inactivation of the alpha-galactosidase locus in carriers of Fabry's disease, Science, 170(3954), 1970, 180-181.

[21] R. Froissart, N. Guffon, M.T. Vanier and R.J. Desnick, Fabry disease: D313Y is an alpha-galactosidase A sequence variant that causes pseudodeficient activity in plasma, Molecular Genetics and Metabolism, 80(3), 2003, 307-314.

[22] D. Elstein, and G. Altarescu, Gal A: Molecular genetics of Fabry disease and genotype-phenotype correlation, in D. Elstein (Ed.), Fabry disease, (New York: Springer, 2010) 3-19.

[23] P.D. Stenson, E.V. Ball, and M. Mort, Human gene mutation database 2003 update, Human Mutation, 21(6), 2003, 577-581.

[24] H.S. Bernstein, D.F. Bishop, K.H. Astrin, R. Kornreich, C.M. Eng, H. Sakuraba, and R.J. Desnick, Fabry disease: six gene rearrangements and an exonic point mutation in the alpha-galactosidase gene, The Journal of Clinical Investigation, 83(4), 1989, 1390-1399.

[25] R. Kornreich, D.F. Bishop, and R.J. Desnick, Alpha-galactosidase A gene rearrangements causing Fabry disease: identification of short direct repeats at breakpoints in an Alu-rich gene, The Journal of Biological Chemistry, 265(14), 1990, 9319-9326.

[26] C. M. Eng, and R.J. Desnick, Molecular basis of Fabry disease: mutations and polymorphisms in the human a-galactosidase A gene, Human Mutation, 3(2), 1994, 103-111.

[27] A.K. Topaloqlu, G.A. Ashley, B. Tong, J. Shabbeer, K.H. Astrin, C.M. Eng, and R.J. Desnick, Twenty novel mutations in the alpha-galactosidae A gene causing Fabry disease, Molecular Medicine, 5(12), 1999, 806-811.

[28] M. Yasuda, J. Shabeer, S.D. Benson, I. Maire, R.M. Burnett, and R.J. Desnick, Fabry disease: characterization of alphagalactosidase A double mutations and the D313Y plasma enzyme pseudodeficiency allele, Human Mutation, 22(6), $2003,486-492$.

[29] S. Ishii, H. Sakuraba, and Y. Suzuki, Point mutations in the upstream region of the $\alpha$-galactosidase A gene exon 6 in an atypical variant of Fabry disease, Human Genetics, 89(1), 1992, 29-32.

[30] T. Okumiya, and H. Sakuraba, Fabry disease (alpha-galactosidase deficiency), Nihon Rinsho, 53(12), 1995, 2952-2959.

[31] C.M. Eng, G.A. Ashley, T.S. Burgert, A.L. Enriquez, M.D. Souza, and R.J. Desnick, Fabry disease: thirty-five mutations in the alpha-galactosidase A gene in patients with classic and variant phenotypes, Molecular Medicine, 3(3), 1997, $174-182$.

[32] D. Rosenthal, Y.H. Lien, D. Lager, L.W. Lai, S. Shang, N. Leung, and F.C. Fervenza, A novel alpha-galactosidase a mutant (M42L) identified in a renal variant of Fabry disease, American Journal of Kidney Diseases, 44(5), 2004, 85-89.

[33] J.S. Mayes, J.B. Scheerer, R.N. Sifers, and M.L. Donaldson, Differential assay for lysosomal alpha-galactosidases in human tissues and its application to Fabry's disease, Clinica chimica acta, 112(2), 1981, 247-251.

[34] A.C. Vedder, G.E. Linthorst, M.J. Van Breemen, J.E. Groener, F.J. Bemelman, A. Strijland, M.M. Mannens, J.M. Aerts, and C.E. Hollak, The Dutch Fabry cohort: diversity of clinical manifestations and Gb3 levels, Journal of Inherited Metabolic Disease, 30(1), 2007, 68-78

[35] K. Mills, P. Morris, P. Lee, A. Vellodi, S. Waldek, E. Young, and B. Winchester, Measurement of urinary CDH and CTH by tandem mass spectrometry in patients hemizygous and heterozygous for Fabry disease, Journal of Inherited Metabolic Disease, 28(1), 2005, 35-48.

[36] F. Breunig, F. Weidemann, M. Beer, A. Eggert, V. Krane, M. Spindler, J. Sandstede, J. Strotmann, and C. Wanner, Fabry disease: diagnosis and treatment, Kidney International Supplement, (84), 2003, 181-185.

[37] W.B. Dobyns, A. Filauro, B.N. Tomson, A.S. Chan, A.W. Ho, and N.T. Ting, Inheritance of most X-linked traits is not dominant or recessive, just X-linked, American Journal of Medical Genetics. Part A, 129(2), 2004, 136-143.

[38] D. Elstein, E. Schachamorov, R. Beeri, and G. Altarescu, X-inactivation in Fabry disease, Gene, 505(2), $2012,266-268$.

[39] A. Abe, S. Gregory, L. Lee, P.D. Killen, R.O. Brady, A. Kulkarny, and J.A. Shayman, Reduction of globotriaos ylceramide in Fabry disease mice by substrate deprivation, The Journal of Clinical Investigation, 105(11), 2000, 1563-1571.

[40] S.C. Jung, I.P. Han, A. Limaye, R. Xu, M.P. Gelderman, P. Zefras, K. Tirumalai, G.J. Murray, M.J. During, R.O. Brady, and P. Qasba, Adeno-associated viral vector mediated gene transfer results in long-term enzymatic and functional correction in multiple organs of fabry mice, Proceedings of the National Academy of Sciences of the United States of America, 98(5), 2001, $2676-2681$.

[41] K. Lee, X. Jin, K. Zhang, L. Copertino, L. Andrews, J. Baker-Malcolm, L. Geagan, H. Qiu, K. Seiger, D. Barngrover, J.M. McPherson, and T. Edmunds, A biochemical and pharmacological comparison of enzyme replacement therapies for the glycolipid storage disorder Fabry disease, Glycobiology, 13(4), 2003, 305-313.

[42] H. Sakuraba, M. Murata-Ohsawa, I. Kawashima, Y. Tajima, M. Kotani, T. Ohshima, Y. Chiba, M. Takashiba, Y. Jigami, T. Fukushige, T. Kanzaki, and K. Itoh, Comparison of the effects of agalsidase alfa and agalsidase beta on cultured human Fabry fibroblasts and Fabry mice, Journal of Human Genetics, 51(3), 2006, 180-188. 
[43] R. Parini, M. Rigoldi, F. Santus, F. Furlan, P. De Lorenzo, G. Valsecchi, D. Concolino, P. Strisciuglio, S. Feriozzi, R. Di Vito, R. Ravaglia, R. Ricci, and A. Morrone, Enzyme replacement therapy with agalsidase alfa in a cohort of Italian patients with AndersonFabry disease: testing the effects with the Mainz Severity Score Index, Clinical Genetics, 74(3), 2008, 260-266.

[44] C. Whybra, E. Miebach, E. Mengel, A. Gal, K. Baron, M. Beck, and C. Kampmann, A 4- year study of the efficacy and tolerability of enzyme replacement therapy with agalsidase alfa in 36 women with Fabry disease, Genetics in Medicine, 11(6), 2009, 441-449.

[45] J.E. Wraith, A. Tylki-Szymanska and N. Guffon, Safety and efficacy of enzyme replacement therapy with agalsidase beta: an international, open-label study in pediatric patients with Fabry disease, The Journal of Pediatrics, 152(4), 2008, 563-570.

[46] C. Siatskas, and J.A. Medin, Gene therapy for Fabry disease, Journal of Inherited Metabolic Disease, 24(2), 2001, 25-41.

[47] M.S. Sands, and B.L. Davidson, Gene therapy for lysosomal storage diseases, Molecular Therapy, 13(5), 2006, 25-41.

[48] M.E. Gelsthorpe, N. Baumann, E. Millard, S.E. Gale, S.J. Langmade, J.E. Schaffer, and D.S. Ory, Niemann-Pick type C1 I1061T mutant encodes a functional protein that is selected for endoplasmic reticulum-associated degradation due to protein misfolding, The Journal of Biological Chemistry, 283(13), 2008, 8229-8236.

[49] O. Motabar, E. Sidransky, E. Goldin, and W. Zheng, Fabry disease- current treatment and new drug development, Current Chemical Genomics, 4, 2010, 50-56.

[50] R.A. Dwek, T.D. Butters, F.M. Platt, and N. Zitzmann, Targeting glycosylation as a therapeutic approach, Nature Reviews Drug Discovery, 1(1), 2002, 65-75.

[51] T. Cox, R. Lachmann, C. Hollak, J. Aerts, S. Van Weely, M. Hrebícek, F. Platt, T. Butters, R. Dwek, C. Moyses, I. Gow, D. Elstein, and A. Zimran, Novel oral treatment of Gaucher's disease with N-butyldeoxynojirimycin (OGT 918) to decrease substrate biosynthesis, Lancet, 355(9214), 2000, 1481-1485.

[52] D. Elstein, C. Hollak, J.M. Aerts, S. Van Weely, M. Maas, T.M. Cox, R.H. Lachmann, M. Hrebicek, F.M. Platt, T.D. Butters, R.A Dwek, and A. Zimran, Sustained therapeutic effects of oral miglustat (Zavesca, N-butyldeoxynojirimycin, OGT 918) in type I Gaucher disease, Journal of Inherited Metabolic Disease, 27(6), 2004, 757-766.

[53] S. Ishii, H.H. Chang, K. Kawasaki, K. Yasuda, H.L. Wu, S.C. Garman, and J.Q. Fan, Mutant alpha-galactosidase A enzymes identified in Fabry disease patients with residual enzyme activity: biochemical characterization and restoration of normal intracellular processing by 1-deoxygalactonojirimycin, The Biochemical Journal, 406(2), 2007, 285-295.

[54] D.P. Germain, and J.Q. Fan, Pharmacological chaperone therapy by active-site specific chaperones in Fabry disease: in vitro and preclinical studies, International Journal of Clinical Pharmacology and Therapeutics, 47, 2009, 111-117.

[55] V. Bernier, M. Lagace, D.G. Bichet, and M. Bouvier, Pharmacological chaperones: potential treatment for conformational diseases, Trends in Endocrinology and Metabolism, 15(5), 2004, 222-228.

[56] J.Q. Fan, and S. Ishii, Active-site-specific chaperone therapy for Fabry disease. Yin and Yang of enzyme inhibitors, The FEBS Journal, 274(19), 2007, 4962-4971.

[57] E.R. Benjamin, R. Khanna, A. Schilling, J.J. Flanagan, L.J. Pellegrino, N. Brignol, Y. Lun, D. Guillen, B.E. Ranes, M. Frascella, R. Soska, J. Feng, L. Dungan, B. Young, D.J. Lockchart, and K.J. Valenzano, Co-administration with the pharmacological chaperone AT1001 increases recombinant human $\alpha$-Galactosidase A tissue uptake and improves substrate reduction in Fabry mice, Molecular Therapy, 20(4), 2012, 717-726.

[58] G. Andreotti, V. Citro, A. De Crescenzo, P. Orlando, M. Cammisa, A. Correra, and M.V. Cubellis, Therapy of Fabry disease with pharmacological chaperones: from in silico predictions to in vitro tests, Orphanet Journal of Rare Diseases, 6(6), 2011, 1-10.

[59] C. Li, J.Z. Robin, M. Cherry, M. Lukason, J.D. Robert, N.S. Yew, and S.H. Cheng, Adenovirus transduced lung as a portal for delivering $\alpha$-Galactosidase A into systemic circulation for Fabry disease, Molecular Therapy, 5(6), 2002, 745-754. 